\title{
Piecewise rational manifold surfaces with sharp features
}

\author{
Giovanni Della Vecchia, Bert Jüttler \\ Johannes Kepler University, Institute of Applied Geometry \\ Linz, Austria
}

\begin{abstract}
We present a construction of a piecewise rational free-form surface of arbitrary topological genus which may contain sharp features: creases, corners or cusps. The surface is automatically generated from a given closed triangular mesh. Some of the edges are tagged as sharp ones, defining the features on the surface. The surface is $\mathcal{C}^{s}$ smooth, for an arbitrary value of $s$, except for the sharp features defined by the user. Our method is based on the manifold construction and follows the blending approach.
\end{abstract}

Keywords. Manifold surface, sharp features, smooth piecewise rational free-form surface, arbitrary topological genus, geometric continuity.

\section{Introduction}

Several approaches for free-form surface modeling exist. The main approaches include geometrically continuous spline surfaces, subdivision surfaces and manifoldbased constructions.

The constructions of geometrically continuous spline surfaces generate collections of piecewise polynomial or rational patches, which are joined together with various degrees of smoothness, e.g., [13-15,17, 18, 20,21]. The available techniques include the use of singular parameterizations and of multisided generalizations of Bézier surfaces.

Subdivision surfaces have been developed into a very valuable tool for freeform surface modeling, see e.g. [19,22]. Their theory - in particular the shape analysis around extraordinary vertices - has made substantial progress during the last years. However, these surfaces do not possess closed-form parametric representation in the vicinity of extraordinary points and it is difficult to achieve higher orders of smoothness.

Another class of methods is based on the notion of a differentiable manifold from differential geometry. This notion has been developed into a construction for smooth surfaces of arbitrary topological genus which are covered by a collection of local parameterizations. The first paper in this direction was authored by Grimm and Hughes [6], and this interesting approach was developed further in a number of publications $[3,4,7,8,16,24]$. In particular $[7,8]$ present a construction of an affine structure for manifold spline surfaces. This construction requires only 
one chart for the evaluation at one point, but it has the disadvantage that in general holes in the mesh are required to deal with models of arbitrary topology.

In some cases, the possibility to define and to describe sharp features on the surface is required in order to obtain adequate results. Therefore, the problem of modeling piecewise smooth free-form surfaces which possess a number of sharp features is of some interest.

So far, most of the available techniques for this solving task are either based on subdivision surfaces or they work directly with triangular meshes, see e.g. $[5,12]$. The two papers $[2,11]$ describe methods to define the path of feature curves at arbitrary locations on the surface. Various other approaches, mostly for surfaces represented by triangular meshes, have been proposed [1, 10,23].

In this paper we present a manifold-based free-form surface construction which can generate sharp features. More precisely, given a closed triangular mesh of arbitrary topological genus, we generate a free-form surface. Some of edges of the mesh can be tagged as "sharp" edges, defining the feature curves on the freeform surface. The surface can achieve any desired order of smoothness, except of course along the feature curves, where it is just continuous. The construction is based on an extension of our recent paper [4], where we constructed a manifold surface by blending together circular charts.

The method presented in this paper preserves the manifold structure (i.e. the parameterized atlas associated with the mesh) and the surface is evaluated following the blending approach. The definition of sharp features in our construction is possible because of the high flexibility which is available in the definition of the transition functions, which are generated by the method of subchart parameterization. This may be an advantage compared to other manifold constructions which are based on more uniform transition functions.

The remainder of the this paper consists of four parts. Section 2 summarizes the method described in [4], which is the starting point for the construction presented in this paper. Section 3 extends the previous results to manifold surfaces with sharp features. The fourth section presents several examples. Finally we conclude this paper.

\section{A construction of rational manifold surfaces}

In order to make this paper self-contained we recall a construction of a rational spline manifold which was presented in [4]. This construction proceeds in three steps. We define (1) charts and subcharts, (2) generate subchart parameterizations, transition functions and the domain, and (3) obtain the manifold surface as a blending-based embedding of the domain.

\subsection{Charts and subcharts}

We consider an oriented triangular mesh $M$ in $\mathbb{R}^{3}$ with $m_{V}$ vertices, where the $i$ th vertex possesses the valency $v(i)$. 
Let $[n(i, j)]_{j=0}^{v(i)-1}$ be the list of neighbours of the $i$ th vertex in counterclockwise order with respect to the orientation of $M$. The second argument of $n$ will be used modulo $v(i)$, i.e., $n(i, j)=n(i, j+k v(i))$ for all $k \in \mathbb{Z}$. Let

$$
\begin{aligned}
& V=\left\{i: i=1, \ldots, m_{V}\right\}, \\
& E=\{\{i, n(i, r)\}: i \in V, r=1, \ldots, v(i)\}, \text { and } \\
& F=\left\{\{i, n(i, r), n(i, r+1)\}: i=1, \ldots, m_{V}, r=1, \ldots, v(i)\right\} .
\end{aligned}
$$

be the sets of vertex, edge and face indices. Note that we use set-valued edge and face indices, hence the order within these indices is not relevant.

For each vertex index $i \in V$, we define an associated chart $C^{i} \subset \mathbb{R}^{2} \times\{i\}$,

$$
C^{i}=\left\{(x, y, i): x^{2}+y^{2} \leq 1\right\}
$$

which is essentially a circular unit disk, centered at the origin. The third coordinate $i$ has been added in order to obtain mutually disjoint charts,

$$
i \neq j \Rightarrow C^{i} \cap C^{j}=\emptyset \text {. }
$$

The chart $C^{i}$ is subdivided into edge and face subcharts and an innermost region, as follows.

- For each edge $\{i, n(i, r)\}$ of the mesh which starts or ends at the $i$ th vertex, the chart $C^{i}$ possesses an edge subchart $C_{n(i, r)}^{i}$.

- For each face $\{i, n(i, r), n(i, r+1)\}$ which shares the $i$ th vertex, the chart $C^{i}$ has a face subchart $C_{n(i, r), n(i, r+1)}^{i}$, where $r=1, \ldots, v(i)$.

- The remaining or innermost part of $C^{i}$ is

$$
\hat{C}^{i}=\overline{C^{i} \backslash\left(\bigcup_{r=1, . ., v(i)} C_{n(i, r)}^{i} \cup \bigcup_{r=1, . ., v(i)} C_{n(i, r), n(i, r+1)}^{i}\right)} .
$$

The generic layout of charts and subcharts is shown in Figure 1, see also [4]. The subcharts are arranged in counterclockwise order along the boundaries of the charts.

The face subcharts are triangular regions with two straight and one circular boundary. They correspond to the overlap of the local parameterizations which are defined by three charts.

The edge subcharts are quadrangular regions with one circular, two straight and one free-form boundary (which is shared with the innermost part of the chart). The overlapping region of the chart $C^{i}$ with another chart $C^{n(i, r)}$, where $(i, n(i, r))$ is an edge of the mesh, is the union of two face subcharts and one edge subchart,

$$
O_{n(i, r)}^{i}=C_{n(i, r-1), n(i, r)}^{i} \cup C_{n(i, r)}^{i} \cup C_{n(i, r), n(i, r+1)}^{i} .
$$

It corresponds to the overlap of the local parameterizations which are defined by two charts. This region has the shape of a biangle with two $\mathcal{C}^{s}$ smooth boundary curves meeting in two vertices, where $s$ is the order of smoothness of the manifold surface. 


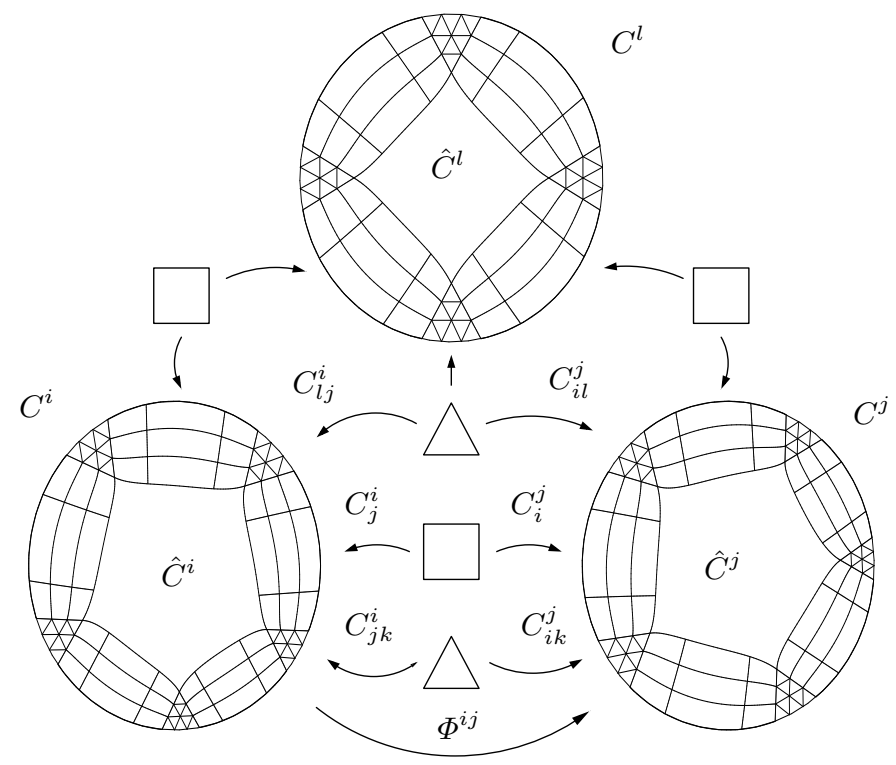

Fig. 1. Charts, subcharts and subchart parameterizations.

\subsection{Transition functions and domain}

We define the transition functions with the help of subchart parameterizations, whose domains are the unit square $\square$ or the standard triangle $\triangle$,

$$
\square=[0,1]^{2} \text { and } \triangle=\{(u, v, w): u \geq 0, v \geq 0, w \geq 0, u+v+w=1\} .
$$

More precisely, we define the following parameterizations.

- For each edge $e=\{i, j\} \in E$ of the triangular mesh we define two edge subchart parameterizations

$$
\phi_{j}^{i}: \square \rightarrow C_{j}^{i} \text { and } \phi_{i}^{j}: \square \rightarrow C_{i}^{j} .
$$

- For each face $f=\{i, j, k\} \in F$ of the triangular mesh, we define three face subchart parameterizations,

$$
\phi_{j k}^{i}: \triangle \rightarrow C_{j k}^{i}, \quad \phi_{k i}^{j}: \triangle \rightarrow C_{k i}^{j} \text { and } \phi_{i j}^{k}: \triangle \rightarrow C_{i j}^{k}
$$

The order of the lower indices is not relevant, $\phi_{j k}^{i}=\phi_{k j}^{i}$.

These mappings are assumed to be $\mathcal{C}^{s}$ smooth, surjective, and orientation preserving. Moreover, they are assumed to be compatible in the following sense:

- For each vertical edge of the unit square, there is exactly one of the two edge subchart parameterizations (9) which maps it into the boundary of the chart. 
- For each edge of the standard triangle, there is exactly one of the three face subchart parameterizations (10) which maps it into the boundary of the chart.

Now we define the transition function (or coordinate transformation) between any pair of charts $C^{i}$ and $C^{j}$ with $\{i, j\} \in E$. Let $\{i, j, k\},\{i, j, l\} \in F$ such that

$$
\begin{aligned}
& \exists r: k=n(i, r-1), j=n(i, r), l=n(i, r+1), \\
& \exists s: l=n(j, s-1), i=n(j, s), k=n(j, s+1) .
\end{aligned}
$$

The transition function $\Phi^{i j}$ maps the overlapping region $O_{j}^{i} \subset C^{i}$ into the overlapping region $O_{i}^{j} \subset C^{j}$,

$$
\Phi^{i j}: O_{j}^{i} \rightarrow O_{i}^{j}: u \mapsto\left\{\begin{array}{lll}
\left(\phi_{l i}^{j} \circ\left(\phi_{l j}^{i}\right)^{-1}\right)(u) & \text { if } & u \in C_{l j}^{i} \\
\left(\phi_{i}^{j} \circ\left(\phi_{j}^{i}\right)^{-1}\right)(u) & \text { if } & u \in C_{j}^{i} \\
\left(\phi_{i k}^{j} \circ\left(\phi_{j k}^{i}\right)^{-1}\right)(u) & \text { if } & u \in C_{j k}^{i}
\end{array} .\right.
$$

These transition functions obey the cocyle condition,

$$
\left(\Phi^{j k} \circ \Phi^{i j}\right)(u)=\Phi^{i k}(u) \quad \text { if } \quad u \in C_{j k}^{i},
$$

since the overlapping region of three charts is parameterized with respect to the common domain $\triangle$. However, the transition functions are not automatically guaranteed to be $\mathcal{C}^{s}$ smooth, where $s$ is the required degree of smoothness of the surface. This is needed in order to obtain a manifold surface which possesses this smoothness. The smoothness of the transition functions has to be ensured by the construction of the subchart parameterizations.

For each chart $C^{i}$, associated with the vertex $i$, we choose the face subchart parameterizations $\phi_{j k}^{i}$ as planar rational Bézier triangles of degree two. The layout of these Bézier triangles along the boundary of the chart is automatically determined by projecting the neighboring vertices into the tangent plane of the vertex $i$.

The edge subchart parameterizations $\phi_{j}^{i}$ are then constructed as rational tensor product patches of degree $(4,4 s+2)$. Using a construction which is based on Möbius transformations and blending we are able to achieve $\mathcal{C}^{s}$ smoothness of the transition functions.

See [4] for more details about the parameterization of face and edge subcharts.

The transition functions $\Phi^{i j}$ define an equivalence relation on the union of the charts $\bigcup_{i \in V} C^{i}$. More precisely, two points $u \in C^{i}$ and $v \in C^{j}$ are considered as equivalent $(\sim)$, if the transition function $\Phi^{i j}$ maps $u$ into $v$,

$$
u \sim v \Longleftrightarrow \exists\{i, j\} \in E: \Phi^{i j}(u)=v .
$$

The domain of the manifold surface with charts $C^{i}$ and transition functions $\Phi^{i j}$ is then obtained by forming the equivalence classes of this relation,

$$
\Omega=\left(\bigcup_{i \in V} C^{i}\right) / \sim
$$


The elements of $\Omega$ are sets containing one, two or three points of different charts.

The face $f=\{i, j, k\} \in F$ of the mesh $M$ corresponds to a subset of $\Omega$ which consists of sets with three elements,

$$
\Omega^{i j k}=\left\{\left\{\phi_{j k}^{i}(t), \phi_{i k}^{j}(t), \phi_{i j}^{k}(t)\right\}: t \in \triangle\right\} \subset \Omega .
$$

The edge $e=\{i, j\} \in E$ of the mesh $M$ corresponds to a subset of $\Omega$ which consists of sets with two elements,

$$
\Omega^{i j}=\left\{\left\{\phi_{j}^{i}(t), \phi_{i}^{j}(t)\right\}: t \in \square\right\} \subset \Omega .
$$

Finally, the $i$ th vertex of the mesh corresponds to a subset which consists of sets with only one element,

$$
\Omega^{i}=\left\{\{u\}: u \in \hat{C}^{i}\right\} \subset \Omega
$$

where $\hat{C}^{i}$ is the innermost part of the chart $C^{i}$.

\subsection{Manifold surface by blending}

The manifold spline surface is generated as an embedding of the domain. For each chart $C^{i}$ we define a geometry function $\mathbf{g}^{i}$ and an influence function $\beta^{i}$.

- The geometry function

$$
\mathbf{g}^{i}: C^{i} \rightarrow \mathbb{R}^{3}:(x, y, i) \mapsto \mathbf{g}^{i}(x, y)
$$

is a vector-valued bivariate quadratic polynomial, which is automatically generated by approximating the neighbours of the $i$ th vertex of the given mesh.

- The influence function $\beta^{i}$ is a suitable power of the circle equation,

$$
\beta^{i}: C^{i} \rightarrow \mathbb{R}:(x, y, i) \mapsto\left(1-x^{2}-y^{2}\right)^{s+1}
$$

where $s$ is the desired order of smoothness of the surface. This defines a bubble function whose first $s$ derivatives vanish along the boundary of $C^{i}$.

Finally we define the embedding of the domain $\Omega$ by blending together the contributions of all charts,

$$
\mathbf{p}: \Omega \rightarrow \mathbb{R}^{3}: u \mapsto \frac{\sum_{u \cap C^{i} \neq \emptyset, i \in V} \beta^{i}\left(u \cap C^{i}\right) \mathbf{g}^{i}\left(u \cap C^{i}\right)}{\sum_{u \cap C^{i} \neq \emptyset, i \in V} \beta^{i}\left(u \cap C^{i}\right)}
$$

Depending on the cardinality of $u$, the point $\mathbf{p}(u)$ is obtained by blending together three, two or one geometry functions.

For all $u \in \Omega^{i j k}$, which correspond to the face $\{i, j, k\}$ of the given mesh $M$, the set of corresponding points on the manifold surface can be parameterized as 
a rational triangular patch with the domain $\triangle$. For all $u \in \Omega^{i j}$, which correspond to the edge $\{i, j\}$ of $M$, the set of corresponding points on the manifold surface can be parameterized as a rational quadrangular patch with domain $\square$. Finally, the innermost parts of the charts can be covered by triangular or quadrangular patches, which again correspond to rational patches on the manifold surface. Consequently we obtain a $C^{s}$ smooth manifold surface, which can be represented as a collection of rational quadrangular and triangular surface patches.

Remark 1. Since the degree of the quadrangular and triangular surface patches is relatively high, it is not recommended to represent them in closed form as Bézier patches. Nevertheless, the surfaces are expressed in explicit form and it is simple to use tools such as automatic differentiation.

\section{Extension to sharp features}

We extend the presented framework in order to model objects with sharp features. Sharp features can be classified as darts, creases and corners (see [9, 12]). Smooth curves along which the surface presents tangent discontinuity are creases. Corners are points where three or more creases meet; a dart is an interior point of the surface where a crease starts or ends. We adapt our construction to all three types.

\subsection{Sharp edges and $k$-vertices}

For any given mesh $M$, the construction presented in the previous section produces a $C^{s}$ smooth surface (provided that no singularities occur) from a given mesh. In certain situations, however, the given mesh is not suitable for a smooth surface generation, and the method generates inadequate results. For instance, this is the case if large dihedral angles between the faces of the triangles are present.

We assume that some of the edges of the mesh are tagged as sharp edges. For instance, one may simply choose all edges where the angle between the incident faces exceeds a certain threshold.

Consider the $i$ th vertex with valency $v(i)$. Depending on the number $k$ of sharp edges which share this vertex, this vertex is classified as a $k$-vertex of the mesh.

$-k=0:$ A $0-$ vertex corresponds to a smooth region on the surface.

$-k=1:$ A 1 -vertex corresponds to a dart on the surface, i.e., to a point where a crease starts or ends.

$-k=2$ : A 2 -vertex corresponds to a segment of a crease on the surface

$-k \geq 3$ : Such a vertex corresponds to a corner of the surface. 


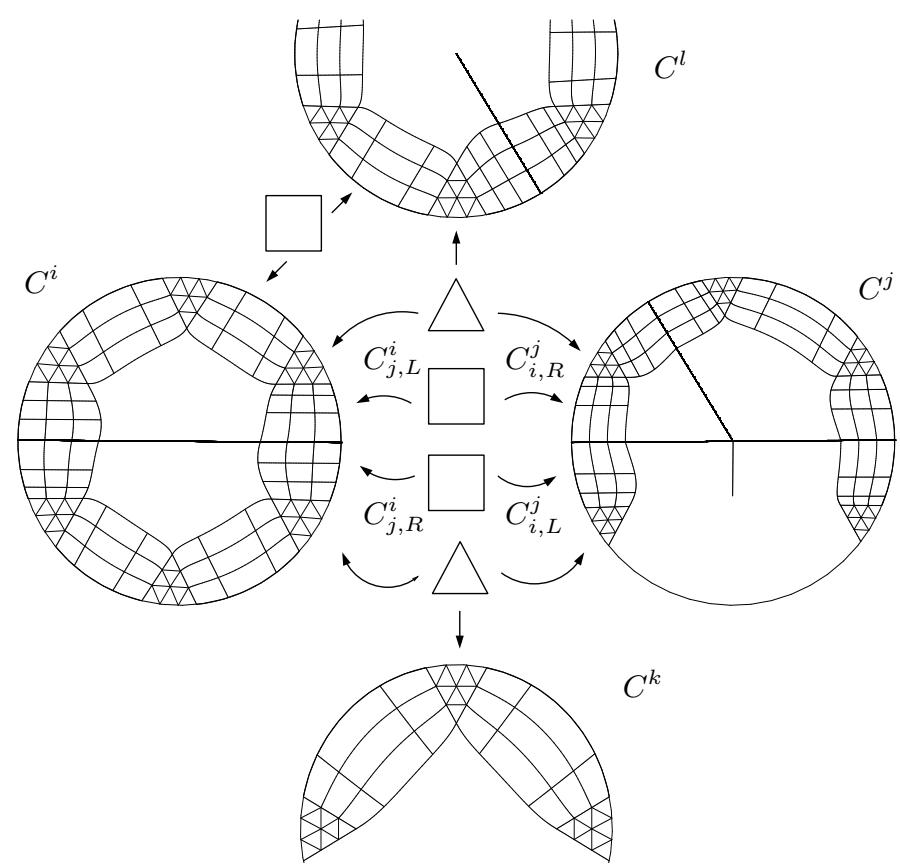

Fig. 2. Definition of $k$-charts and subcharts for different values of $k$. The chart $C^{i}$ (center, left) is a 2-chart.

\section{$3.2 k$-charts and their subcharts}

If the $i$ th vertex is a $k$-vertex, then the chart associated with it will be called a $k$-chart. We describe how to adapt the constructions of the subcharts and of the subchart parameterizations for $k \neq 0$. The case $k=0$ is dealt with as in [4].

The construction of the $k$-charts and of its subcharts consists of three steps (cf. Fig. 2).

- Step 1: Face subcharts and face subchart parameterizations. The face subcharts $C_{n(i, r), n(i, r+1)}^{i}$ are constructed using the method described in [4]. We choose the layout of the face subcharts based on the geometry of the mesh in the vicinity of the $i$ th vertex. More precisely, we consider the projection of the neighborhood of into the estimated tangent plane and use it to choose certain geometric parameters controlling the layout of edge and face subcharts. The face subcharts are then parameterized by quadratic rational Bézier triangles with one circular and two straight line boundaries.

- Step 2: Central point $\mathbf{c}^{i}$ and edge lines. We choose a central point $\mathbf{c}^{i} \in C^{i}$, where the edge lines will meet. For each sharp edge $\{i, n(i, r)\}$ emanating from the $i$ th vertex, we choose an edge line $E_{n(i, r)}^{i}$ which connects $\mathbf{c}^{i}$ with a point on the boundary $\partial C^{i}$ of the chart, where this point is located between the face subcharts $C_{n(i, r-1), n(i, r)}^{i}$ and $C_{n(i, r), n(i, r+1)}^{i}$. 
In our implementation, the central point is simply the center of the circle, and the edge lines are straight line segments. An exception is made for $k=2$. In that case, we get only two edge lines, which are supposed to meet smoothly at the central point. In that case we choose both edge lines as a straight line segment connecting two points on the boundary $\partial C^{i}$, and the central point as its midpoint.

- Step 3: Edge semi-subcharts. For each sharp edge $\{i, n(i, r)\}$, the edge subchart $C_{n(i, r)}^{i}$ is subdivided into a left and a right semi-subchart $C_{n(i, r), L}^{i}$ and $C_{n(i, r), R}^{i}$ with associated parameterizations. The left (resp. the right) edge semi-subchart shares one boundary arc with $C_{n(i, r-1), n(i, r)}^{i}$ (resp. with $\left.C_{n(i, r), n(i, r+1)}^{i}\right)$ and one with the innermost part $\hat{C}^{i}$. The remaining two boundary arcs are segments of the edge line $E_{n(i, r)}^{i}$ and of the chart boundary $\partial C^{i}$. The boundary arcs of the two semi-subcharts cover approximately one third of the edge line $E_{j}^{i}$.

The remaining edge subcharts (which correspond to non-sharp edges) are generated as in the case $k=0$, see [4].

The parameterization of the two semi-subcharts is described in the next section.

\subsection{Parameterization of semi-subcharts}

The edge semi-subcharts are parameterized by mappings whose domain is the unit square $\square=[0,1]^{2}$. These two mappings will be called left and right edge semi-subchart parameterization

$$
\phi_{j, L}^{i}: \square \rightarrow C_{j, L}^{i} \quad \text { and } \quad \phi_{j, R}^{i}: \square \rightarrow C_{j, R}^{i} .
$$

The transition functions between two charts are defined as in the smooth case, see (13). These transition functions need to be $C^{s}$ smooth everywhere except along the edge lines, where $s$ is the desired smoothness of the manifold surface. This is obtained by ensuring that the two edge semi-subcharts satisfy the following conditions.

- They have a $\mathcal{C}^{s}$ smooth joint with the neighboring face subcharts which are reparameterized as tensor-product patches.

- They have a $\mathcal{C}^{0}$ joint along the edge line $E_{j}^{i}$.

- Their outer boundary is contained in the boundary $\partial C^{i}$.

The construction of the edge semi-subchart parameterizations is obtained with the help of Möbius transformations, similar to the case of edge subchart parameterizations (cf. [4]). We describe in more detail the construction of a right edge semi-subchart, see also Fig. 3. The other case is dealt with analogously.

Let $\mu$ be a Möbius transformation that maps the unit circle into the real axis, which is computed in the same way as in [4]; let $\rho$ be the reparameterization

$$
\rho: \square \rightarrow \triangle:(r, s) \mapsto(r,(1-r) s,(1-r)(1-s))
$$




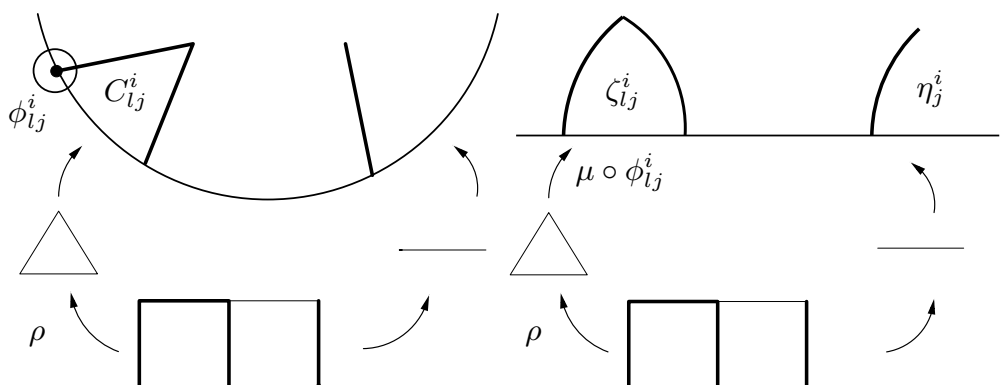

(a)

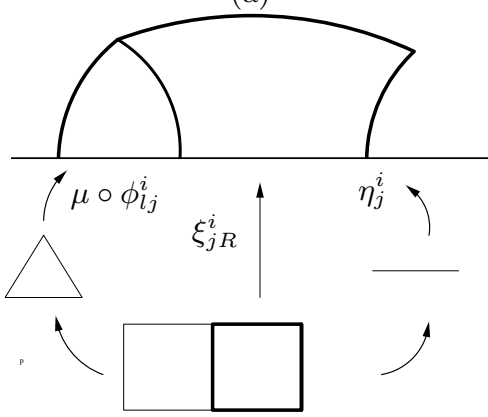

(c) (b)

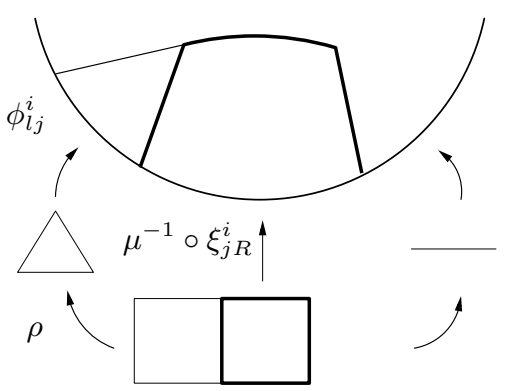

(d)

Fig. 3. Parameterization of a right edge semi-subchart. (a) The neighbouring face subchart is represented as a degenerate quadrangular patch, (b) applying the Möbius transformation, (c) construction of a tensor-product patch, (d) the inverse Möbius transformation gives the desired parameterization.

that represents a triangular patch as a degenerate tensor product patch, with the edge $r=1$ collapsing into a singular point.

1. The face subchart parameterization $\phi_{l j}^{i}$ is a quadratic Bézier triangle. By composing it with $\rho$ we obtain a degenerate tensor-product patch of degree $(1,2)$ (see [4]). Finally it is composed with $\mu$ and this gives the rational tensor-product patch

$$
\zeta_{l j}^{i}=\mu \circ \phi_{l j}^{i} \circ \rho
$$

of degree $(2,4)$ which parameterizes the image $\mu\left(C_{l j}^{i}\right)$. We consider a linear parameterization of the segment of the edge line $E_{j}^{i}$, where the two semisubcharts are to be joined. By composing this parameterization with $\mu$ we obtain the curve $\eta_{j}^{i}$.

2. We create a tensor-product patch $\xi_{j R}^{i}$ which possesses a $\mathcal{C}^{s}$ smooth joint with $\zeta_{l j}^{i}$ and $\mathcal{C}^{0}$ joint with $\eta_{j}^{i}$. This patch can be chosen as a rational tensorproduct patch of degree $(2, s+1)$. 


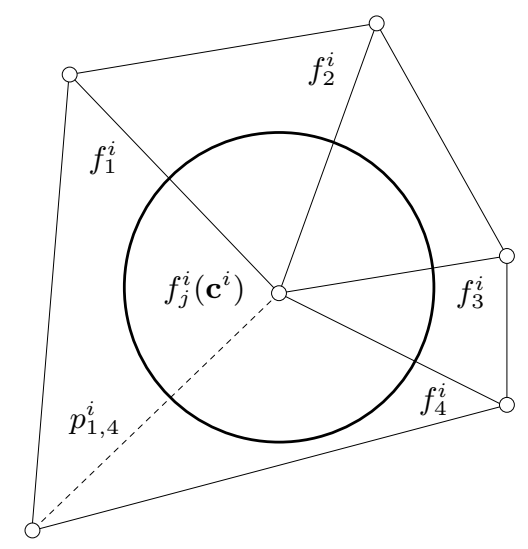

Fig. 4. Example of geometry function in the planar case as piecewise function consisting of Bézier triangles. Since the angle between $f_{1}^{i}$ and $f_{4}^{i}$ is greater than $\pi$, the phantom edge $p_{1,4}^{i}$ is introduced, defining two Bézier triangles joined with $\mathcal{C}^{d}$ continuity.

3. We apply the inverse Möbius transformation in order to get the desired edge semi-subchart parameterization

$$
\phi_{j, R}^{i}=\mu^{-1} \circ \xi_{j, R}^{i} .
$$

This gives a rational tensor product patch of degree $(4,2 s+2)$.

\subsection{Feature lines and geometry functions}

Finally we have to generate geometry functions which take the edge lines into account. Let the chart $C^{i}$ be a $k$-chart with $k$ edge lines $E_{j}^{i}$. If $k=0$, then the geometry function is computed as in the smooth case. Otherwise we assume that - for each edge line - a feature curve $f_{j}^{i}: E_{j}^{i} \rightarrow \mathbb{R}^{3}$ is given. All feature curves share the common point $f_{j}^{i}\left(\mathbf{c}^{i}\right)$. Moreover, if $k=2$, then the two feature curves have a $\mathcal{C}^{s}$ smooth joint.

The feature curves can either be specified by the user, or they can be automatically generated by approximating the sharp edges of the mesh by smooth curves.

The geometry function $\mathbf{g}^{i}: C^{i} \rightarrow \mathbb{R}^{3}$ of the $k$-chart is now chosen as a continuous piecewise polynomial function with $k$ pieces which respects the feature curves, i.e.,

$$
\left.\mathbf{g}^{i}\right|_{E_{j}^{i}}=f_{j}^{i}
$$

holds for all sharp edges $\{i, j\}$. It is $C^{s}$ smooth, except for the edge lines.

For example, each geometry function $\mathbf{g}^{i}$ can be defined as a piecewise function consisting of $k$ Bézier triangles, corresponding to the $k$ feature lines $f_{j}^{i}$. These Bézier triangles are defined such that the control points along the boundaries, 


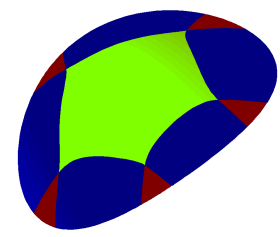

$k=0$

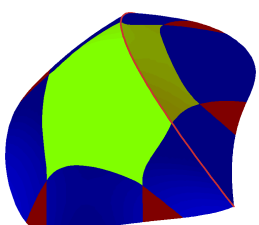

$k=2$

valency 6

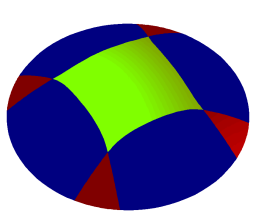

$k=0$

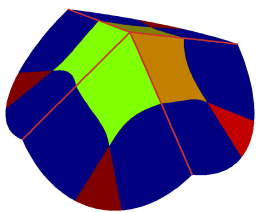

$k=4$

Fig. 5. Geometry functions for different valencies and for different values of $k$. The different colors visualize the different subcharts.

i.e. the feature lines, coincide. In case the angle between two feature lines $f_{j}^{i}$ and $f_{k}^{i}$ exceeds $\pi$, one can introduce a phantom edge $p_{j k}^{i}$ between these feature lines and define the piece of $\mathbf{g}^{i}$ between $f_{j}^{i}$ and $f_{k}^{i}$ as two Bézier triangles joined with a certain $\mathcal{C}^{d}$ continuity along $p_{j k}^{i}$. Figure 4 shows an example of a piecewise defined geometry function in the planar case.

In our implementation, if $k \geq 3$, then the feature curves are segments and the geometry are piecewise linear. If $k=2$, then we choose cubic feature curves and piecewise cubic geometry functions. In all other cases we choose the geometry functions as in [4].

Figure 5 shows the geometry functions for $k$-charts with different valencies.

\section{Examples}

We present three examples for surfaces which have been generated via the proposed method. All surfaces can be represented as a collection of rational surface patches which form $C^{2}$ smooth manifold surfaces, except for the sharp features. The construction was implemented in Maple, and the surfaces were visualized using PovRay, where each quadrangular resp. triangular surface patch was rendered using 50 resp. 25 triangles.

Example 1 (see Fig. 6). The triangular mesh consists of 8 vertices and 12 faces. We defined two loops of edge curves which meet in the top vertex. Consequently, the mesh defines six 2 -charts, one 4 -chart and one 0 -chart (the bottom one). The figure shows the results of the construction with and without sharp features. In addition, we also show the result which is obtained by defining a 1-chart, which leads to a dart feature on the surface.

Example 2 (see Fig. 7) This surface has been generated from a star-shaped polyhedron, consisting of 18 vertices and 32 faces. We create a crease feature by defining a closed curve of four sharp edges, which leads to four 2-charts and 14 $0-$ charts. 


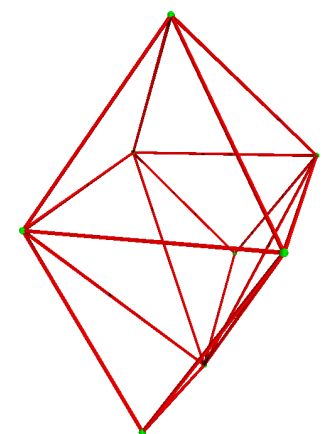

(a)

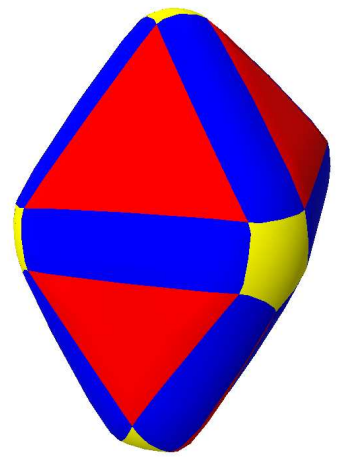

(d)

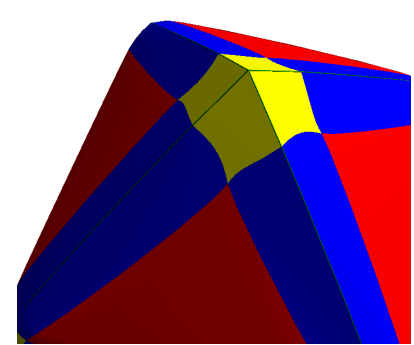

(g)

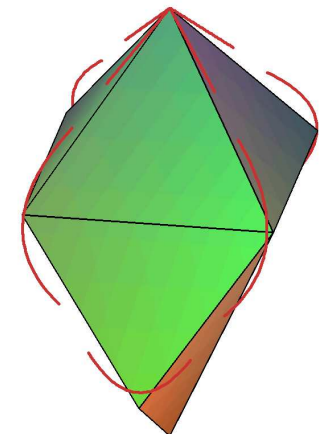

(b)

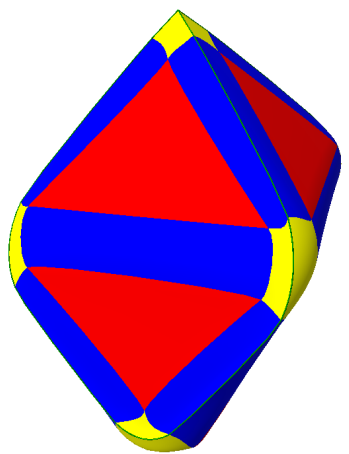

(e)

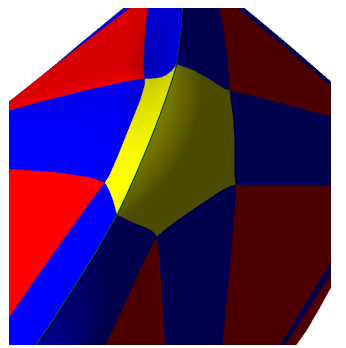

(h)

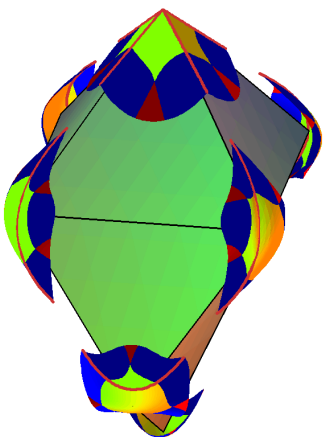

(c)

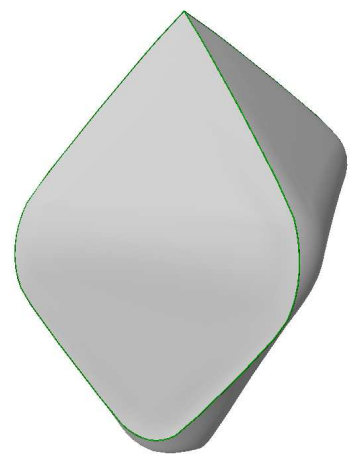

(f)

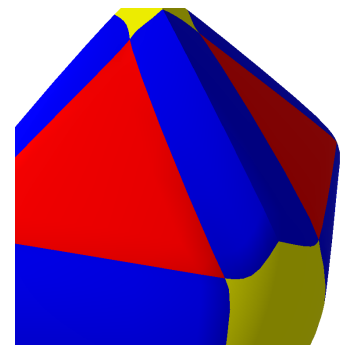

(i)

Fig. 6. Example 1: Manifold surface generated from a mesh with 8 vertices and two loops of sharp edges. The mesh (a), feature curves (b), geometry functions (c), a smooth model obtained with smooth geometry functions (d), the model with sharp edges (e,f), and details of the features $(\mathrm{g}, \mathrm{h})$ are shown. The last plot (i) shows the effect of a 1-chart which generates a dart feature on the surface. The different colors in $(\mathrm{d}, \mathrm{e}, \mathrm{g}, \mathrm{h})$ visualize the contributions of the different subcharts. 


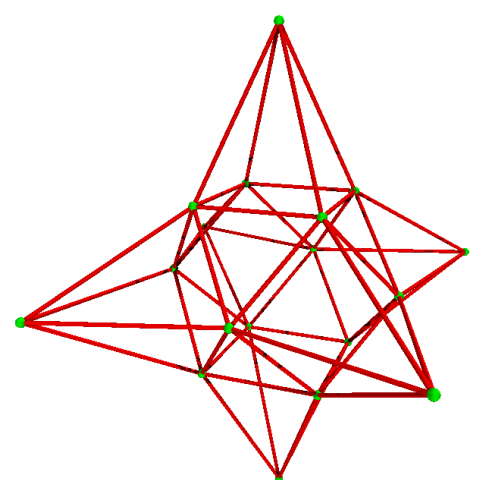

(a)

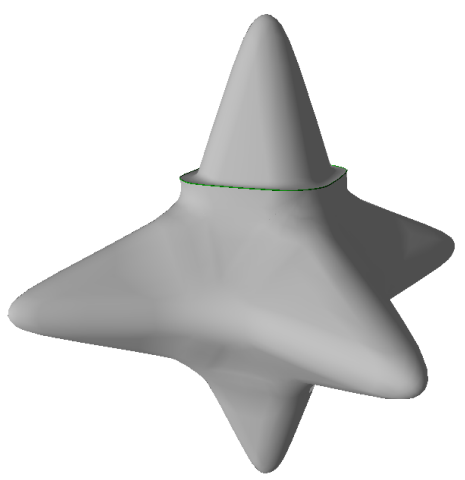

(c)

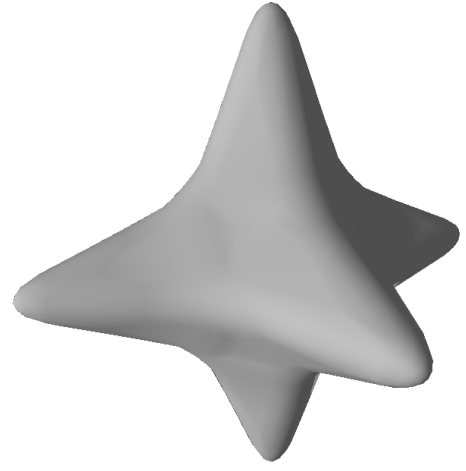

(b)

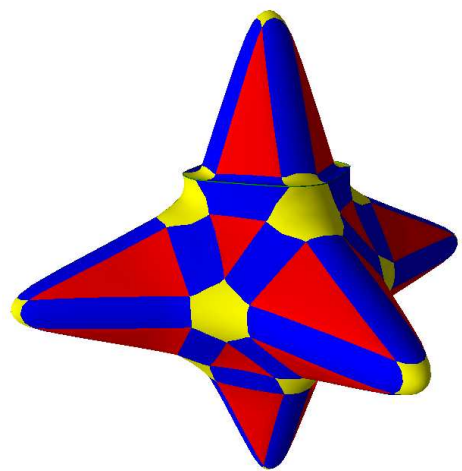

(d)

Fig. 7. Example 2: A manifold spline surface (b) obtained from a star-shaped polyhedron (a) and a surface which was obtained defining a loop of sharp edges (c,d).

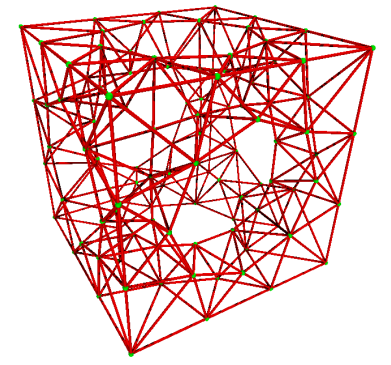

(a)

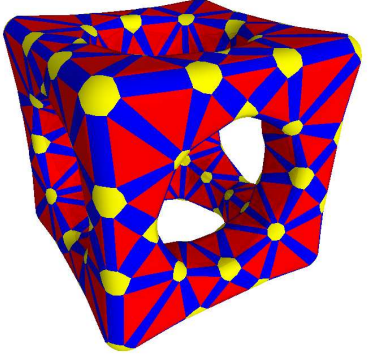

(b)

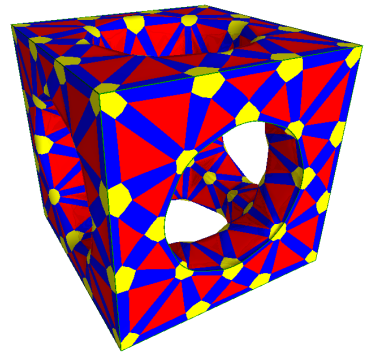

(c)

Fig. 8. Example 3: A smooth manifold surface (b) obtained from a mesh of a hollow cube (a) and a surface with sharp features along the outer edges and around one of the circles (c). 
Example 3 (see Fig. 8). The final examples has been obtained from a mesh describing a hollow cube with 112 vertices and 240 faces. The sharp features correspond to the edges of the cube, which define 12 creases and 8 corners, and to the circles in the faces. Here we modified only one of these circles.

\section{Conclusions}

We presented a new construction of piecewise rational free-form surfaces with the possibility to define sharp features on it, based on the manifold construction already presented in [4]. Starting from a triangular mesh, where some edges are tagged as sharp edges, the algorithm generates automatically a surface, which can achieve any order of smoothness, except for the sharp features.

The definition of sharp features on the surface does not modify the manifold representation and the transition functions between overlapping charts, which keep the $\mathcal{C}^{s}$ smoothness everywhere except along the feature lines. The surface can be represented as a collection of quadrangular and triangular rational patches.

Future research is required concerning the optimal choice of the geometry functions, in order to obtain a better visual fairness of the manifold surfaces. Also, the analysis of manifold constructions which generate spline surfaces of lower degree is of potential interest.

Acknowledgment This research has been supported by the Austrian Science Fund (FWF) in the frame of the FSP S092 "Industrial geometry", subproject 2.

\section{References}

1. M. Attene, B. Falcidieno, J. Rossignac, and M. Spagnuolo. Sharpen and bend: recovering curved sharp edges in triangle meshes produced by feature-insensitive sampling. IEEE Transactions on Visualization and Computer Graphics, 11(2):181192, 2005.

2. H. Biermann, I. Martin, D. Zorin, and F. Bernardini. Sharp features on multiresolution subdivision surfaces. In In Proc. Pacific Graphics, pages 140-149, 2001.

3. J. Cotrina-Navau and N. Pla-Garcia. Modeling surfaces from meshes of arbitrary topology. Computer Aided Geometric Design, 17(7):643-671, 2000.

4. G. Della Vecchia, B. Jüttler, and M.-S. Kim. A construction of rational manifold surfaces of arbitrary topology and smoothness from triangular meshes. Computer Aided Geometric Design, 25(9):801-815, 2008.

5. T. DeRose, M. Kass, and T. Truong. Subdivision surfaces in character animation. In Proc. SIGGRAPH, pages 85-94, New York, NY, USA, 1998. ACM.

6. C. Grimm and J. Hughes. Modeling surfaces of arbitrary topology using manifolds. In Proc. Siggraph, pages 359-368, New York, 1995. ACM Press.

7. X. Gu, Y. He, M. Jin, F. Luo, H. Qin, and S.-T. Yau. Manifold splines with single extraordinary point. In Proc. Solid and Physical modeling, pages 61-72, New York, 2007. ACM Press.

8. X. Gu, Y. He, and H. Qin. Manifold splines. In Proc. Solid and Physical Modeling, pages 27-38, New York, 2005. ACM Press. 
9. H. Hoppe, T. DeRose, T. Duchamp, M. Halstead, H. Jin, J. McDonald, J. Schweitzer, and W. Stuetzle. Piecewise smooth surface reconstruction. In Proc. SIGGRAPH, pages 295-302, New York, 1994. ACM.

10. A. Hubeli and M. Gross. Multiresolution feature extraction from unstructured meshes. In IEEE Visualization, 2001.

11. A. Khodakovsky and P. Schröder. Fine level feature editing for subdivision surfaces. In Proc. Shape Modelling Appl., pages 203-211, New York, 1999. ACM.

12. R. Ling, W. Wang, and D. Yan. Fitting sharp features with Loop subdivision surfaces. Comput. Graph. Forum, 27(5):1383-1391, 2008.

13. C. Loop and T. D. DeRose. A multisided generalization of Bézier surfaces. ACM Trans. Graph., 8(3):204-234, 1989.

14. C. Loop and T. D. DeRose. Generalized B-spline surfaces of arbitrary topology. In Proc. SIGGRAPH, pages 347-356, New York, 1990. ACM Press.

15. Charles Loop. Smooth spline surfaces over irregular meshes. In Proc. SIGGRAPH, pages 303-310, New York, 1994. ACM Press.

16. J. Cotrina Navau, N. Pla Garcia, and M. Vigo Anglada. A generic approach to free form surface generation. In Proc. Solid Modeling and Applications, pages 35-44, New York, 2002. ACM Press.

17. J. Peters. $C^{2}$ free-form surfaces of degree $(3,5)$. Computer Aided Geometric Design, 19:113-126, 2002.

18. J. Peters. Geometric continuity. In G. Farin, J. Hoschek, and M.-S. Kim, editors, Handbook of Computer Aided Geometric Design. Elsevier, 2002.

19. J. Peters and U. Reif. Subdivision surfaces. Springer, 2008.

20. H. Prautzsch. Freeform splines. Computer Aided Geometric Design, 14(3):201-206, 1997.

21. U. Reif. TURBS - topologically unrestricted rational B-splines. Constructive Approximation, 14:57-77, 1998.

22. M. A. Sabin, T.J. Cashman, U. H. Augsdorfer, and N. A. Dodgson. Bounded curvature subdivision without eigenanalysis. In R. Martin et al., editors, Mathematics of surfaces XII, volume 4647 of Lecture Notes in Computer Science. Springer, 2007.

23. H. Yang and B. Jüttler. Evolution of T-spline level sets for meshing non-uniformly sampled and incomplete data. The Visual Computer, 24:435-448, 2008.

24. L. Ying and D. Zorin. A simple manifold-based construction of surfaces of arbitrary smoothness. Proc. Siggraph, ACM Transactions on Graphics, 23(3):271-275, 2004. 